\title{
Honokiol suppresses proliferation and induces apoptosis via regulation of the miR-21/PTEN/PI3K/AKT signaling pathway in human osteosarcoma cells
}

\author{
JIEXIANG YANG $^{1 *}$, YONGGEN ZOU $^{2}$ and DIANMIN JIANG ${ }^{1}$ \\ ${ }^{1}$ Department of Orthopedics, The First Affiliated Hospital of Chongqing Medical University, Chongqing 400016; \\ ${ }^{2}$ Department of Orthopedics, The Second Affiliated Hospital of Southwest Medical University, \\ Luzhou, Sichuan 646000, P.R. China
}

Received March 6, 2017; Accepted November 15, 2017

DOI: $10.3892 /$ ijmm.2018.3433

\begin{abstract}
Honokiol (HNK) is a small biphenolic compound, which exerts antineoplastic effects in various types of cancer. However, the mechanism underlying the antitumor effects of HNK in osteosarcoma (OS) cells is not yet fully understood. Emerging evidence has indicated that microRNAs (miRNAs/miRs) serve key roles in numerous pathological processes, including cancer. It has previously been reported that Chinese medicinal herbs harbor anticancer properties via modulating miRNA expression. Therefore, the present study aimed to determine whether HNK could suppress OS cell growth by regulating miRNA expression. The 3-(4,5-dimethylthiazol-2-yl)-2,5-diphenyltetrazolium bromide (MTT) assay and flow cytometric analysis were used to evaluate the cell proliferation and apoptosis in human OS cells after treatment with HNK, respectively. The results demonstrated that HNK inhibits proliferation and induces apoptosis of human OS cells in a dose-dependent manner. Furthermore, HNK-induced apoptosis was characterized by upregulation of proapoptotic proteins, including cleaved-caspase-3, cleaved-poly (ADP-ribose) polymerase and B-cell lymphoma 2 (Bcl-2)-associated $\mathrm{X}$ protein, and downregulation of the anti-apoptotic protein Bcl-2. Reverse transcription-quantitative polymerase chain reaction (RT-qPCR) verified that HNK was able to induce aberrant expression of miRNAs in human OS cells, and miR-21 was one of the miRNAs that was most significantly downregulated. To further investigate miR-21 function, the present study validated that HNK reduces miR-21 levels in a dose-dependent manner. In addition, restoration of miR-21 expression abrogated the suppressive effects of
\end{abstract}

Correspondence to: Dr Dianmin Jiang, Department of Orthopedics, The First Affiliated Hospital of Chongqing Medical University, 1 Youyi Road, Chongqing 400016, P.R. China

E-mail: dianminjiang@yeah.net

Key words: honokiol, osteosarcoma, microRNA-21, phosphatase and tensin homolog/phosphoinositide 3-kinase/protein kinase B signaling
HNK on OS cells. Luciferase assay and western blot analysis identified that miR-21 inhibits the expression of phosphatase and tensin homolog (PTEN) by directly targeting its 3'-UTR. Notably, HNK was able to suppress the phosphoinositide 3-kinase (PI3K)/protein kinase B (AKT) signaling pathway; however, it was reactivated by miR-21 overexpression. Taken together, these data indicated that HNK may inhibit proliferation and induce apoptosis of human OS cells by modulating the miR-21/PTEN/PI3K/AKT signaling pathway. Therefore, miR-21 may be considered a potential therapeutic target for the treatment of osteosarcoma with HNK.

\section{Introduction}

Osteosarcoma (OS) is the most frequent primary malignant bone tumor, which is commonly diagnosed in children and young adolescents, with a male predominance (1). OS is highly aggressive and primarily metastasizes to the lung (2). Surgical tumor resection and multi-agent chemotherapy are the main current therapeutic strategies used to treat OS. It has previously been reported that chemotherapy may increase the 5-year survival rate for localized disease by $>50 \%$ compared with surgery alone. Conversely, patients diagnosed with metastases exhibit a poor prognosis, with a 5-year survival rate of 20-30\% following surgical resection and/or radiotherapy $(3,4)$. Furthermore, currently approved agents exhibit severe side effects $(3,4)$; therefore, the development of a novel agent with increased efficiency and reduced toxicity in OS treatment is required.

Honokiol (HNK) is a biphenolic compound extracted from the magnolia tree, which has been used to treat anxiety, thrombotic stroke and gastrointestinal symptoms in traditional Chinese and Japanese medicine (5). HNK has long been known to exert antimicrobial (6), anti-inflammatory (7) and antiangiogenic $(8,9)$ effects. Increasing evidence has revealed that HNK exerts antineoplastic functions in various types of cancer, including angiosarcoma (8), colorectal carcinoma (10), breast cancer (11) and gastric cancer (12). Furthermore, HNK may trigger apoptotic pathways that result in mitochondrial dysfunction (13), influence retinoblastoma function and E2F transcription factor 1 transcriptional activity (14), and suppress the phosphoinositide 3-kinase 
(PI3K)/mammalian target of rapamycin (mTOR) pathway (15). However, the molecular mechanism underlying the anticancer effects of HNK on OS cells remains to be elucidated.

MicroRNAs (miRNAs/miRs) are a class of small (19-24 nucleotide) noncoding RNAs that mediate post-transcriptional regulation of target genes by suppressing translation or promoting RNA degradation. miRNAs have crucial functions in various biological and pathological processes, including cellular proliferation, differentiation, apoptosis and carcinogenesis (16). In recent years, it has been demonstrated that some natural products are able to control tumor-suppressive and oncogenic miRNAs, including curcumin (diferuloylmethane), which inhibits hepatocellular cancer cell proliferation via modulating miRNA expression (17). Furthermore, previous studies have reported that Chinese medicinal herbs exert antitumor effects by modulating miRNA expression (18,19). Zhang et al demonstrated that HNK suppresses bladder tumor growth by inhibiting the enhancer of zeste homolog 2/miR-143 axis (20). Avtanski et al also revealed that HNK rescued leptin-induced tumor progression by suppressing the Wnt1-metastasis associated $1-\beta$-catenin signaling pathway in a miR-34a-dependent manner (11). Therefore, it may be hypothesized that HNK inhibits proliferation and induces apoptosis, via the modulation of miRNA expression, in human OS cells.

The present study investigated the effects of HNK on OS tumor growth inhibition and explored the underlying molecular mechanisms. The results indicated that HNK may inhibit growth and promote apoptosis of human OS cells in a dose-dependent manner. Furthermore, the results verified that HNK induces aberrant expression of miRNAs in human OS cells, and miR-21 suppresses phosphatase and tensin homolog (PTEN) by directly targeting its 3'-untranslated region (3'-UTR). Notably, the results indicated that HNK blocks the PI3K/protein kinase B (AKT) signaling pathway by inhibiting miR-21 expression in human OS cells. Collectively, these results suggested that the molecular mechanism by which HNK induces apoptosis was modulated by the miR-21/PTEN/PI3K/AKT axis in human OS cells.

\section{Materials and methods}

Reagents and cell culture. HNK was obtained from the National Institute for the Control of Pharmaceutical and Biological Products (Beijing, China). HNK was dissolved in $10 \mu \mathrm{M}$ dimethyl sulfoxide (DMSO) and was maintained at $4^{\circ} \mathrm{C}$. The human OS cell lines Saos-2 and MG-63 were obtained from the American Type Culture Collection (Manassas, VA, USA) and were grown in Dulbecco's modified Eagle's medium (DMEM; Gibco; Thermo Fisher Scientific, Inc., Waltham, MA, USA) supplemented with $10 \%$ fetal bovine serum (FBS; Gibco; Thermo Fisher Scientific, Inc.), $50 \mathrm{U} / \mathrm{ml}$ penicillin and $50 \mu \mathrm{g} / \mathrm{ml}$ gentamicin (both Sigma-Aldrich; Merck KGaA, Darmstadt, Germany), $2.5 \mu \mathrm{g} / \mathrm{ml}$ amphotericin B, $1 \%$ glutamine and $2 \%$ HEPES at $37^{\circ} \mathrm{C}$ in a humidified incubator containing $5 \% \mathrm{CO}_{2}$.

3-(4,5-Dimethylthiazol-2-yl)-2,5-diphenyltetrazolium bromide (MTT) assay. The MTT assay was used to investigate the antiproliferative effects of HNK on OS cells. Briefly, $1 \times 10^{4}$ cells were seeded into 96-well plates overnight. After treatment with 1-100 $\mu \mathrm{M} \mathrm{HNK}$ for $24 \mathrm{~h}$ at $37^{\circ} \mathrm{C}$, the cells were washed with PBS and incubated for $48 \mathrm{~h}$ at $37^{\circ} \mathrm{C}$ in fresh medium. The cells in the control group were only treated with $100 \mu \mathrm{l}$ DMSO for $24 \mathrm{~h}$ at $37^{\circ} \mathrm{C}$. Subsequently, $20 \mu 15 \mathrm{mg} / \mathrm{ml}$ MTT (Sigma-Aldrich; Merck KGa) solution was added to each well and incubated at $37^{\circ} \mathrm{C}$ for an additional $4 \mathrm{~h}$. The supernatant was then discarded and $150 \mu 1$ DMSO was added to each well. Finally, absorbance of the samples was measured at $490 \mathrm{~nm}$ using a microplate reader (Sunrise ${ }^{\mathrm{TM}}$; Tecan Group Ltd., Männedorf, Switzerland).

Apoptosis analysis. Flow cytometric analysis was used to detect cell apoptosis. Briefly, the cells were treated with 1-100 $\mu \mathrm{M}$ HNK for $24 \mathrm{~h}$ at $37^{\circ} \mathrm{C}$, after which $5 \times 10^{5}$ cells were obtained from the culture and were washed with cold PBS. The cells in the control group were only treated with $100 \mu$ l DMSO for $24 \mathrm{~h}$ at $37^{\circ} \mathrm{C}$. Cell apoptosis was evaluated using the Annexin V/propidium iodide (PI) staining kit (BioVision, Inc., Milpitas, CA, USA) according to the manufacturer's protocol. Flow cytometry was conducted at the Flow Cytometry Core Facility at Cedars-Sinai Medical Center (Los Angeles, CA, USA) using FACScan (BD Biosciences, San Jose, CA, USA). Data were analyzed using the Cell Quest program version 6.0 (FACScan; BD Biosciences).

Western blot analysis. Cells were lysed as described previously (21). Subsequently, a bicinchoninic acid protein assay kit (Pierce; Thermo Fisher Scientific, Inc.) was used to measure protein concentration. Total proteins $(60 \mu \mathrm{g})$ were separated by $10 \%$ SDS-PAGE (Sigma-Aldrich; Merck $\mathrm{KGaA}$ ) and were then transferred onto polyvinylidene difluoride (PVDF) membranes (BD Biosciences). After blocking with $5 \%$ non-fat milk at room temperature for $1 \mathrm{~h}$, PVDF membranes were incubated with primary antibodies (dilution 1:500) for $2 \mathrm{~h}$ at room temperature and followed with a horseradish peroxidase conjugated secondary antibody (dilution 1:1,000) for $1 \mathrm{~h}$ at room temperature. Mouse anti-PTEN (sc-7974), mouse anti-AKT (sc-6546) and mouse anti-p-AKT (Ser473; sc-33437) primary antibodies were purchased from Santa Cruz Biotechnology, Inc. (Dallas, TX, USA). Rabbit anti-mTOR (39408), rabbit anti-p-mTOR (32199), rabbit antip70S6K (32505), rabbit anti-p-p70S6K (66134) and $\beta$-actin (79467) antibodies were purchased from Abcam (Cambridge, MA, USA). Antibodies of apoptosis-associated proteins were as follows: cleaved PARP (ab32064), Bax (ab25901), cleaved caspase-3 (ab13847) and Bcl-2 (ab32503), purchased from Abcam. The corresponding rabbit anti-mouse (315-065-003) and goat anti-rabbit (305-065-003) secondary antibodies were purchased from Jackson ImmunoResearch Laboratories, Inc. (West Grove, PA, USA). Subsequently, protein bands were scanned on X-ray film using the enhanced chemiluminescence detection system (PerkinElmer, Inc., Waltham, MA, USA). AlphaImager software version 2000 (ProteinSimple, San Jose, CA, USA) was used to measure relative intensity of each band on the blots. Measurements were conducted independently at least three times with similar results.

Reverse transcription-quantitative polymerase chain reaction $(R T-q P C R)$. Total cellular RNA was extracted using ZR RNA MicroPrep ${ }^{\mathrm{TM}}$ kit (Zymo Research Corp., Irvine, CA, 
USA) according to the manufacturer's protocol. RNA concentration was measured using a spectrophotometer (Eppendorf, Hamburg, Germany). The high capacity cDNA synthesis kit (Applied Biosystems; Thermo Fisher Scientific, Inc.) was used to synthesize cDNA using miRNA-specific primers according to the manufacturer's protocol. The primers for miR-188-5p, miR-202, miR-623, miR-21, miR-532-5p, miR-628-3p and the internal control RNU44 gene were obtained from Ambion (Thermo Fisher Scientific, Inc.). The primers were as follows: miR-188-5p forward, 5'-TGTGGCTATCTTGCTGCCC-3' and reverse, 5'-GAGT CATTCTCCTTCCCACC-3'; miR-202 forward, 5'-TTAGG CCAGATCCTCAAAGAAG-3' and reverse, 5'-ATAGGAAA AAGGAACGGCGG-3'; miR-623 forward, 5'-ATCCCTT GCAGGGGCTGTTGGGT-3' and reverse, 5'-GCCAGCAC AGAATTAATACGAC-3'; miR-21 forward, 5'-TAGCTTA TCAGACTGATGTTGA-3' and reverse, 5'-GCCAGCA CAGAATTAATACGAC-3'; miR-532-5p forward, 5'-GCCCA TGCCTTGAGTGTAG-3' and reverse, 5'-GTGCGTGTC GTGGAGTCG-3'; miR-628-3p forward, 5'-GGGGGATG CTGACATATTTAC-3' and reverse, 5'-CAGTGCGTG TCGTGGAGT-3'; RNU44 forward, 5'-CCTGGATGATG ATAGCAAATGC-3' and reverse, 5'-GAGCTAATTA AGACCTTCATGTT-3'. qPCR was conducted using TaqMan Gene Expression assay (Applied Biosystems; Thermo Fisher Scientific, Inc.) on an Applied Biosystems 7500 real-time PCR machine (Applied Biosystems; Thermo Fisher Scientific, Inc.). The PCR reaction was performed at $95^{\circ} \mathrm{C}$ for $5 \mathrm{~min}$ followed by $40 \mathrm{cycles}$ of $95^{\circ} \mathrm{C}$ for $30 \mathrm{sec}, 60^{\circ} \mathrm{C}$ for $30 \mathrm{sec}$, and $72^{\circ} \mathrm{C}$ for $30 \mathrm{sec}$. The $2^{-\Delta \Delta \mathrm{Cq}}$ method was used to analyze relative miRNA expression (22). All reactions were performed in triplicate.

Transfection assay. Saos-2 and MG-63 $\left(5 \times 10^{3}\right)$ cells were seeded into each well of 6-well plates, maintained in DMEM containing $10 \% \mathrm{FBS}$, and treated with either control vehicle (DMSO) or HNK. Subsequently, the cells were transfected with miR-21 mimics or miR-21 inhibitor (Guangzhou RiboBio Co., Ltd., Guangzhou, China) at a final concentration of $50 \mu \mathrm{M}$ using Lipofectamine 2000 reagent (Invitrogen; Thermo Fisher Scientific, Inc.) according to the manufacturer's protocol. Cells were collected $48 \mathrm{~h}$ post-transfection. RT-qPCR was used to confirm that miR-21 expression was specifically upregulated/knocked down following transfection with mimics/inhibitor (Fig. 1). Subsequently, OS cell proliferation and apoptosis were assessed by MTT assay and flow cytometric analysis, respectively.

Luciferase assay. The potential binding site between PTEN and miR-21 was identified using TargetScan (http:/www.targetscan.org). The miR-21 mimics/inhibitor and corresponding negative control (NC) were synthesized by Guangzhou RiboBio Co., Ltd. Wild-type (wt) PTEN-3'-UTR and mutant (mut) PTEN-3'-UTR containing the putative binding site of miR-21 were established and cloned into the firefly luciferase-expressing vector pMIR-REPORT (Ambion; Thermo Fisher Scientific, Inc.). For the luciferase assay, Saos- 2 cells at a density of $2 \times 10^{5} /$ well were seeded into 24-well plates and were co-transfected with $0.8 \mu \mathrm{g}$ pMIR-PTEN-3'-UTR or pMIR-PTEN-mut-3'-UTR and
$50 \mathrm{nM}$ miR-21 mimic/inhibitor or corresponding NC using Lipofectamine 2000 reagent (Invitrogen; Thermo Fisher Scientific, Inc.). A total of $48 \mathrm{~h}$ post-transfection, luciferase activity was measured using the dual-light luminescent reporter gene assay (Applied Biosystems; Thermo Fisher Scientific, Inc.). Each experiment was repeated at least three times in independent experiments. The ratio of Renilla luciferase to firefly luciferase was calculated for each well.

Choice of differentially expressed miRNAs list using heat map analysis. We obtained the microarray date from Gene Expression Omnibus (GEO, http://www.ncbi.nlm.nih.gov/ geo/), and the GEO accession no. is GSE85871. Observations with adjusted P-values $\geq 0.05$ were removed, and thus excluded from further analysis. The heat map of the miRNAs most obvious differences was created using a method of hierarchical clustering by GeneSpring GX, version 7.3 (Agilent Technologies, Santa Clara, CA, USA).

Statistical analysis. All statistical analyses were performed using SPSS 14.0 software (SPSS, Inc., Chicago, IL, USA). Each experiment was repeated at least three times. Numerical data are presented as the mean \pm SD. For numerical variables, the results were evaluated by the Student's t-test (comparison between 2 groups) or one way ANOVA to make multiplegroup comparisons followed by the post hoc Tukey's test. $\mathrm{P}<0.05$ was considered to indicate a statistically significant difference.

\section{Results}

HNK inhibits growth of human OS cells. To investigate the antiproliferative effects of HNK on OS cells, Saos-2 and MG-63 cells were treated with various concentrations of HNK for $24 \mathrm{~h}$, and the MTT assay was used to evaluate cell viability. The results indicated that treatment with $1-100 \mu \mathrm{M}$ HNK reduced cell viability of Saos-2 and MG-63 cells in a dose-dependent manner (Fig. 2A and B). The half maximal inhibitory concentration $\left(\mathrm{IC}_{50}\right)$ values of $\mathrm{HNK}$ were $37.85 \mu \mathrm{M}$ in Saos-2 and 38.24 $\mu \mathrm{M}$ in MG-63 cells. Similar $\mathrm{IC}_{50}$ values of HNK were detected in human Saos-2 and MG-63 OS cells.

HNK induces apoptosis of human OS cells. It has been widely reported that HNK may induce apoptosis of various malignant cell types $(9,23)$. To examine HNK-induced apoptosis of OS cells, the cells were analyzed by Annexin V-PI staining following treatment with HNK. The results demonstrated that the proportion of apoptotic cells was markedly increased following HNK treatment compared with in the control group $(\mathrm{P}<0.01)$. Furthermore, following treatment with 10 or $40 \mu \mathrm{M} \mathrm{HNK}$, the number of apoptotic cells increased in a dose-dependent manner (Fig. 2C and D). With regards to apoptotic induction, Saos-2 and MG-63 had similar results (Fig. 2D). To further explore the apoptotic mechanism, the intracellular apoptotic signaling pathway was investigated in OS cells following treatment with various concentrations of HNK. The results revealed that the protein expression levels of cleaved-caspase-3, cleaved-PARP and Bax were significantly upregulated, and Bcl-2 was significantly downregulated following HNK treatment. Furthermore, HNK regulated 

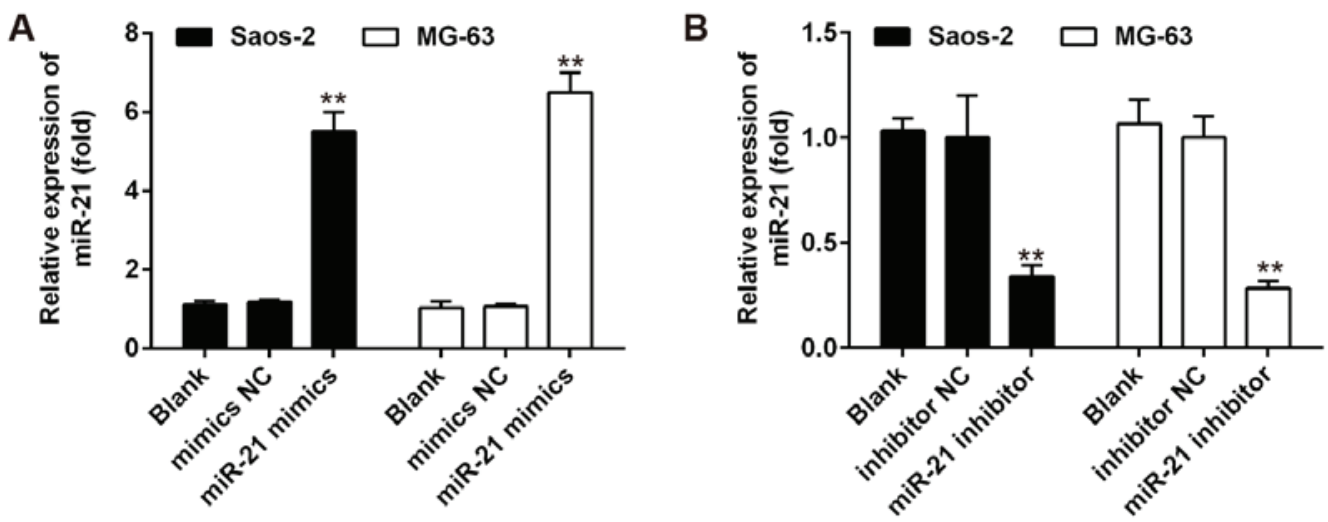

Figure 1. miR-21 expression in Saos-2 or MG-63 cells was detected post-transfection with (A) miR-21 mimics or (B) miR-21 inhibitor. ${ }^{* *} \mathrm{P}<0.01$ vs. the Blank group. miR-21, microRNA-21; NC, negative control.

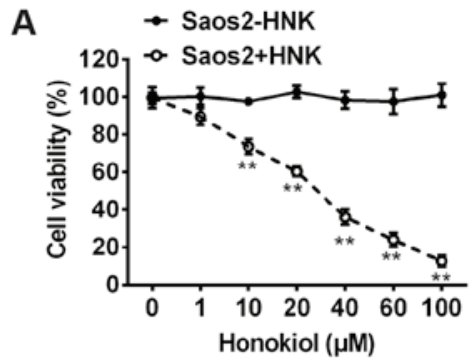

C

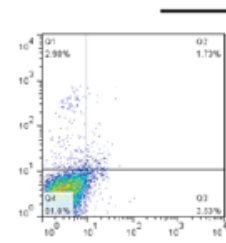

HNK $(\mu \mathrm{M}) \quad 0$

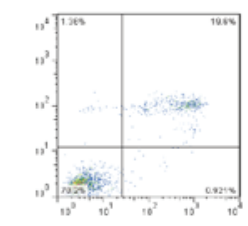

10
B

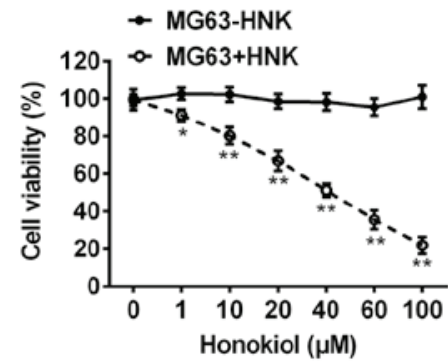

D

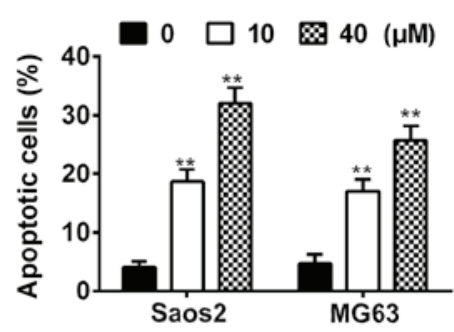

MG63

E

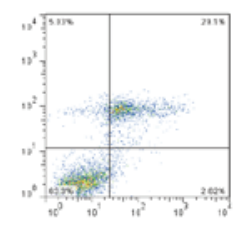

40

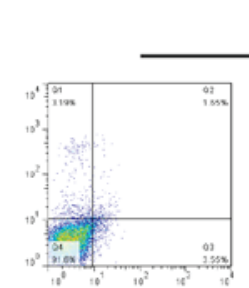

HNK $(\mu \mathrm{M}) \quad 0$

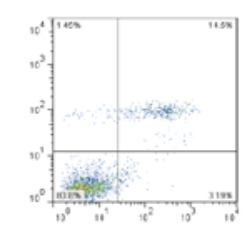

10

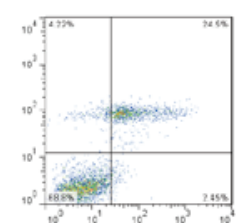

40

Saos-2
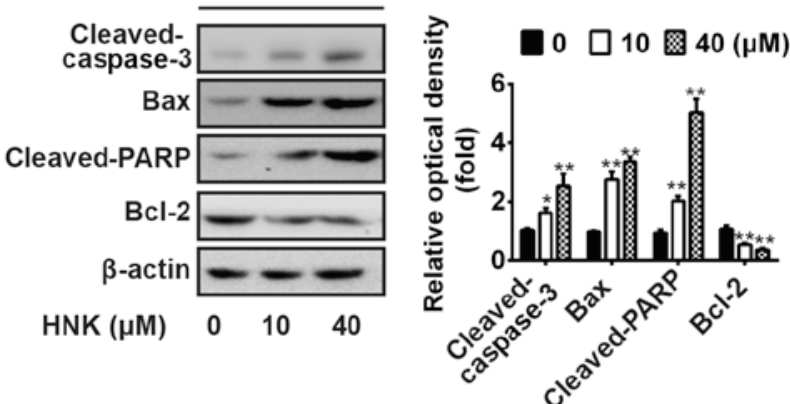
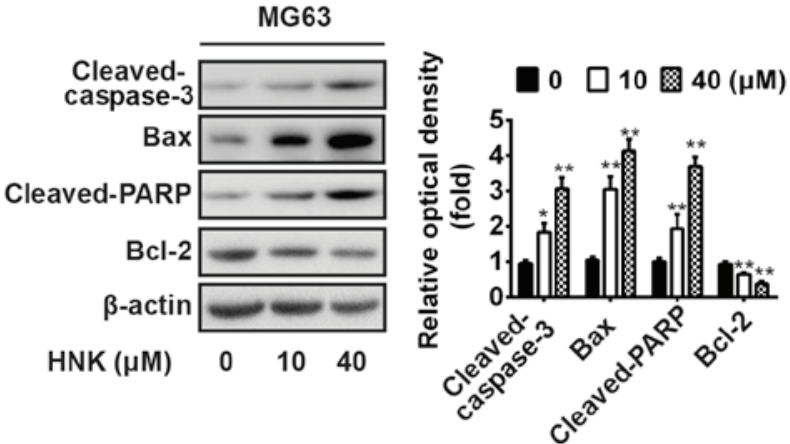

Figure 2. HNK inhibits proliferation and induces apoptosis of human OS cells. (A and B) Human Saos-2 and MG-63 OS cells were treated with or without 1-100 $\mu \mathrm{M}$ HNK for $24 \mathrm{~h}$, and the 3-(4,5-dimethylthiazol-2-yl)-2,5-diphenyltetrazolium bromide assay was conducted to assess viability. (C) Saos-2 and MG-63 cells were treated with or without 10 or $40 \mu \mathrm{M}$ HNK for $24 \mathrm{~h}$, and apoptosis was measured using flow cytometry. (D) Proportion of apoptotic cells was quantified in three independent experiments. (E) Saos-2 and MG-63 cells were treated with or without 10 or $40 \mu \mathrm{M}$ of $\mathrm{HNK}$ for $24 \mathrm{~h}$, and the protein expression levels of cleaved-caspase-3, cleaved-PARP, Bax and Bcl-2 were analyzed by western blot analysis. Representative data from one of three individual experiments with similar results are presented. Data are presented as the mean \pm standard deviation of three independent experiments. ${ }^{*} \mathrm{P}<0.05$ and ${ }^{* *} \mathrm{P}<0.01$ vs. $0 \mu \mathrm{M}$ group. Bax, Bcl-2-associated X protein; Bcl-2, B-cell lymphoma 2; HNK, honokiol; OS, osteosarcoma; PARP, poly (ADP-ribose) polymerase.

these protein expression levels in a dose-dependent manner in human OS cells (Fig. 2E). These data suggested that HNK may induce apoptosis of human OS cells by activating the intracellular apoptotic signaling pathway.
HNK induces miRNA aberrant expression in human OS cells. A recent study revealed that some miRNAs were upregulated in the T24 human bladder cancer cell line following treatment with $9.6 \mu \mathrm{g} / \mathrm{ml} \mathrm{HNK}(20)$. Microarray data obtained from 

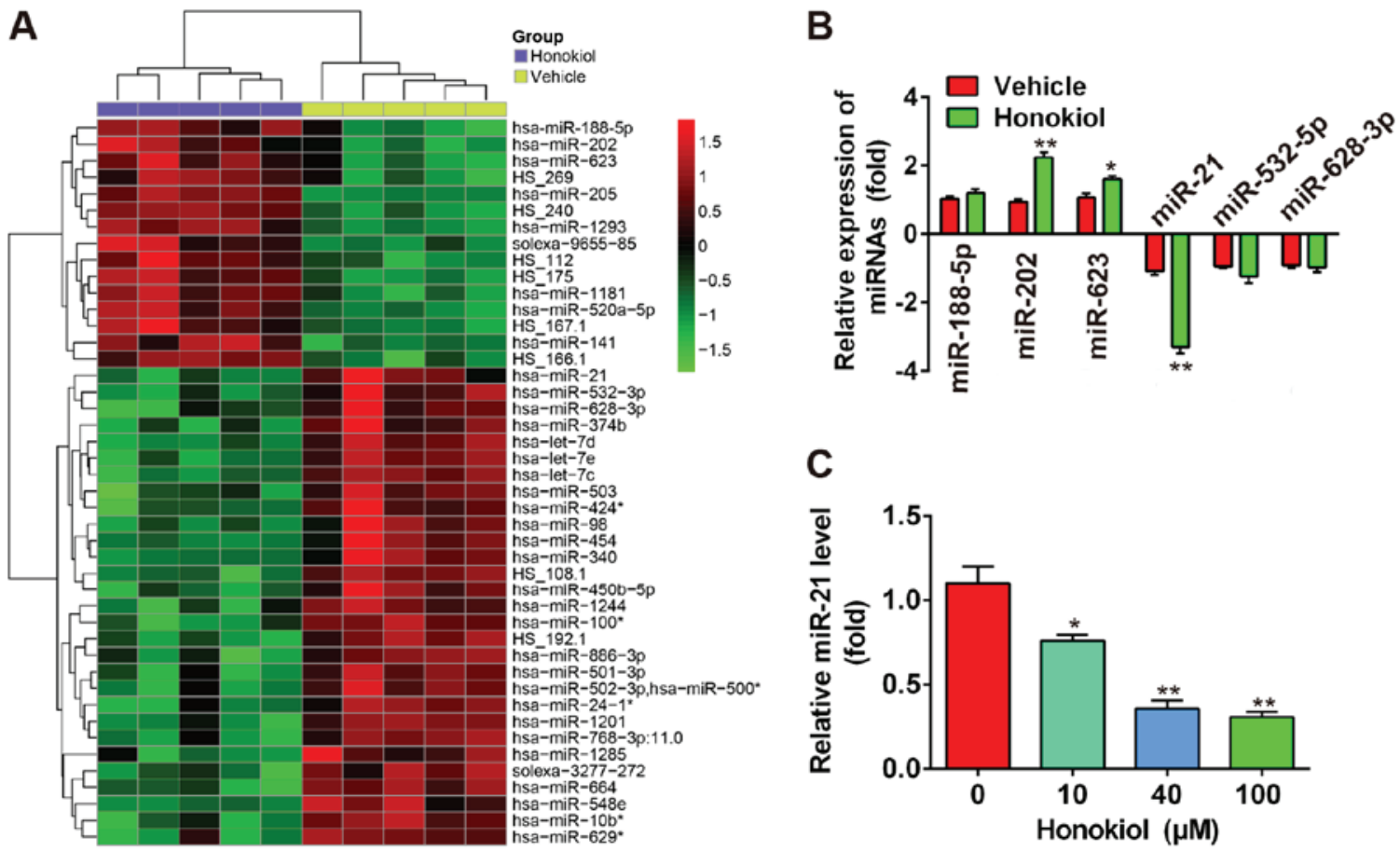

Figure 3. HNK induces aberrant miRNA expression in human OS cells. (A) Microarray data obtained from the Gene Expression Omnibus database (accession no. GSE85871). Heat map shows significant expression alterations of 45 miRNAs in a breast cancer cell line treated with HNK or vehicle. Green represents low expression and red represents high expression. A color change from green to red indicates upregulation and a color change from red to green indicates downregulation. (B) Based on these microarray data, six miRNAs (miR-188-5p, miR-202 and miR-623 were the most significantly upregulated; miR-21, miR-532-5p and miR-628-3p were the most markedly downregulated) were selected and verified by RT-qPCR in OS cells treated with or without HNK. (C) Saos-2 cells were treated with or without 10-100 $\mu \mathrm{M}$ HNK for $24 \mathrm{~h}$, and RT-qPCR was performed to detect the expression levels of miR-21. Data are presented as the mean \pm standard deviation. "P<0.05 and ${ }^{* * *} \mathrm{P}<0.01$ vs. the vehicle group. HNK, honokiol; miRNA/miR, microRNA; OS, osteosarcoma; RT-qPCR, reverse transcription-quantitative polymerase chain reaction.

the Gene Expression Omnibus (GEO) database (accession no. GSE85871, http:/www.ncbi.nlm.nih.gov/geo/query/acc. cgi?acc=GSE85871) indicated that HNK resulted in aberrant expression of miRNAs in a breast cancer cell line (Fig. 3A). To determine whether HNK also induces aberrant expression of miRNAs in human OS cells, six miRNAs (miR-188-5p, miR-202 and miR-623 were the most significantly upregulated; miR-21, miR-532-5p and miR-628-3p were the most significantly downregulated) were selected based on the microarray data, and were verified by RT-qPCR. The results indicated that miR-202 and miR-623 were markedly upregulated, and miR-21 was significantly downregulated in human Saos-2 OS cells following HNK treatment $(\mathrm{P}<0.01)$, whereas miR-188-5p, miR-532-5p and miR-628-3p were not significantly different compared with in the vehicle group (Fig. 3B). miR-21 has previously been reported to be associated with cell apoptosis and proliferation (24). Therefore, the present study further investigated the function of miR-21 in human OS cells. Saos-2 cells were treated with 10-100 $\mu \mathrm{M}$ HNK for $24 \mathrm{~h}$ and the expression levels of miR-21 were determined by RT-qPCR. The results demonstrated that HNK reduced miR-21 levels in a dose-dependent manner in human OS cells (Fig. 3C). These results indicated that HNK may exert antitumor effects via modulating miR-21 expression in human OS cells.

Overexpression of miR-21 rescues the suppressive effects of HNK on OS cells. The present study revealed that miR-21 was downregulated in Saos-2 cells following HNK treatment.
Furthermore, mounting evidence has confirmed that miR-21 may serve a crucial role in regulating the expression of gene products involved in phenotypic characteristics of cancer cells, including cell proliferation, apoptosis and cell cycle $(24,25)$. Therefore, it may be hypothesized that HNK suppresses cell growth and induces apoptosis of OS cells by modulating miR-21 expression. Saos-2 and MG-63 cells were transfected with miR-21 mimics following HNK treatment for 24, 48 and $72 \mathrm{~h}$ (HNK + miR-21 group); treatment with vehicle (vehicle group) or HNK alone (HNK group) served as negative and positive control groups, respectively. Subsequently, cell proliferation in each group was investigated by MTT assay, the results indicated that HNK significantly suppressed cell proliferation; however, the suppressive effects of HNK on OS cells were significantly rescued post-transfection with miR-21 mimics $(\mathrm{P}<0.01$; Fig. 4A and B). To further validate these results, cell apoptosis was measured by flow cytometry. As expected, the proportion of apoptotic cells was significantly decreased in the HNK + miR-21 group compared with in the HNK group in Saos-2 and MG-63 cells ( $\mathrm{P}<0.01$; Fig. $4 \mathrm{C}$ and $\mathrm{D})$. These data indicated that HNK may exert suppressive effects on human OS cells via downregulating miR-21.

miR-21 suppresses PTEN expression by directly targeting its 3 '-UTR. Previous studies have reported that miR-21 may post-transcriptionally suppress PTEN expression in numerous human cancer cells, including lung cancer and esophageal cancer cells $(25,26)$; however, whether PTEN is a direct target of miR-21 in human OS cells remains to be further 
A

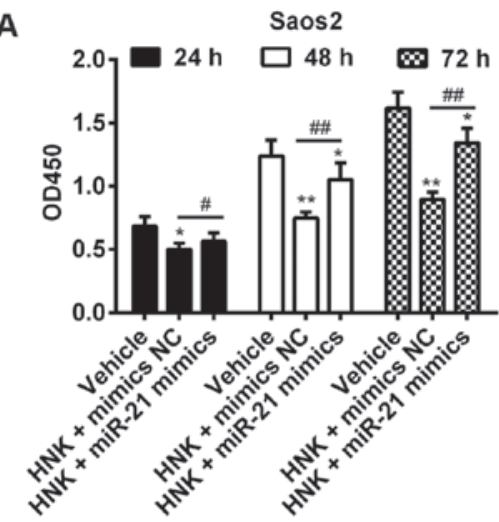

C

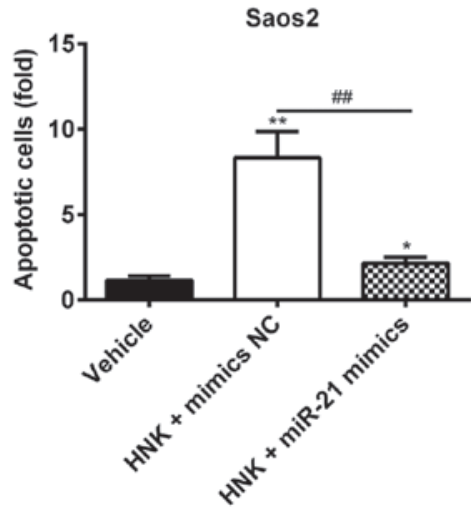

B

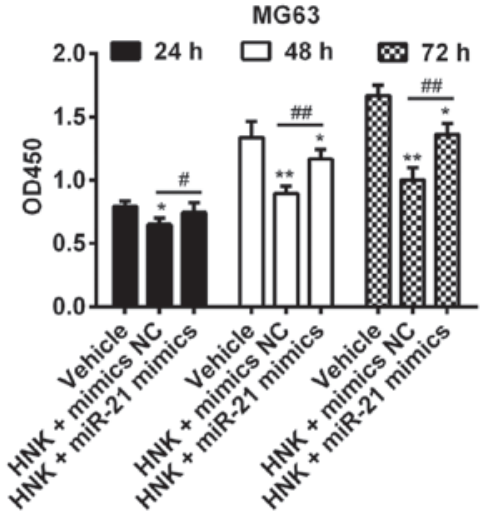

D

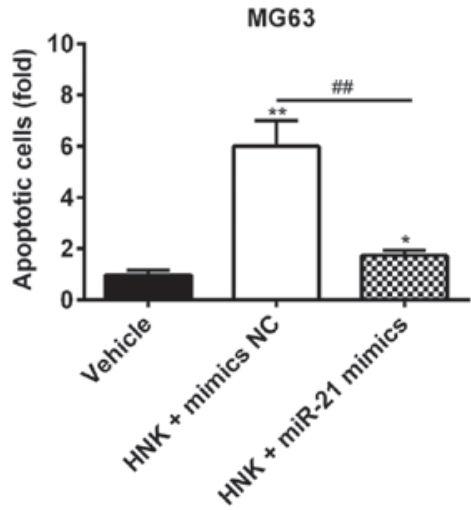

Figure 4. Overexpression of miR-21 rescues the suppressive effects of HNK on OS cells. (A and B) Saos-2 and MG-63 cells were transfected with miR-21 mimics following HNK treatment for 24, 48 and $72 \mathrm{~h}$ (HNK + miR-21 mimics group); treatment with vehicle (vehicle group) or HNK alone (HNK + mimics NC group) served as negative and positive control groups, respectively. Cell proliferation in each group was determined using the 3-(4,5-dimethylthiazol-2-yl)-2,5-diphenyltetrazolium bromide assay. (C and D) Saos-2 and MG-63 cells were transfected with miR-21 mimics following HNK treatment for 48 h, and cell apoptosis was determined using flow cytometric analysis. Data are presented as the mean \pm standard deviation of three independent experiments. ${ }^{*} \mathrm{P}<0.05$, ${ }^{* *} \mathrm{P}<0.01$ vs. the vehicle group. ${ }^{\#} \mathrm{P}<0.05,{ }^{\# \#} \mathrm{P}<0.01$ vs. HNK + mimics NC group. HNK, honokiol; miR-21, microRNA-21; OD, optical density; OS, osteosarcoma.

elucidated. In the present study, luciferase-reporter plasmids containing wt or mut type 3'-UTR segments of PTEN were constructed (Fig. 5A). The reporters were cotransfected alongside miR-21 mimics/inhibitor or NC into Saos-2 cells, after which luciferase activity was measured. The results demonstrated that miR-21 mimic significantly suppressed luciferase activity compared with mimic NC; however, the miR-21 inhibitor markedly enhanced luciferase activity compared with inhibitor NC in the presence of wt 3'-UTR $(\mathrm{P}<0.01$; Fig. 5B). In addition, miR-21 did not affect luciferase activity of the reporter vector containing mut PTEN-3'-UTR (Fig. 5B). These findings indicated that miR-21 inhibits PTEN by directly targeting PTEN-3'-UTR. To further confirm that PTEN levels are modulated by miR-21, the Saos- 2 human OS cell line was transfected with miR-21 mimic/inhibitor or $\mathrm{NC}$, and the protein expression levels of PTEN were determined using western blot analysis. The results indicated that miR-21 inhibited PTEN expression in OS cells compared with the NC (Fig. 5C). To further verify whether PTEN expression was modulated by HNK, Saos-2 cells were treated with 10-100 $\mu \mathrm{M}$ HNK for $24 \mathrm{~h}$ and PTEN expression was measured by western blotting. The results demonstrated that HNK enhanced PTEN expression in a dose-dependent manner in human OS cells (Fig. 5D).

HNK suppresses the PI3K/AKT signaling pathway via modulating miR-21 expression in human OS cells. It has previously been reported that the PI3K/AKT signaling pathway serves a critical role in cell survival, and exerts protective effect against tumorigenesis-associated apoptosis in cancer cells (27). Furthermore, PI3K and AKT are negatively modulated by PTEN, which is a key molecule in various diseases that modulates cell proliferation, survival, apoptosis and metabolism. A recent study revealed that miR-21 may mediate proliferation, apoptosis, migration, invasion and cell cycle progression in human esophageal cancer cells by targeting key proteins of the PTEN/PI3K/AKT signaling pathway (25). Therefore, the present study aimed to determine whether HNK-mediated miR-21 modulation regulates the PI3K/AKT signaling pathway in OS cells. Saos-2 and MG-63 cells were transfected with or without miR-21 mimics following treatment with or without $\mathrm{HNK}$, and western blot analysis was used to determine the expression levels of p-AKT, p-mTOR and p-p70S6K, which are major components of the PI3K/AKT signaling pathway (28). The results indicated that the expression levels of AKT, mTOR and p70S6K were significantly downregulated following HNK treatment compared with mock vehicle-treated cells or miR-21 transfection in Saos-2 and MG-63 cells; however, the expression levels of these proteins were significantly upregulated in HNK-treated OS cells post-transfection with miR-21 mimics compared with transfection without miR-21 mimics $(\mathrm{P}<0.01$; Fig. 6). These results demonstrated that HNK may suppress the PI3K/AKT signaling pathway in human OS cells; however, it could be activated by miR-21 overexpression. Taken together, these data suggested that HNK suppresses the PI3K/AKT 


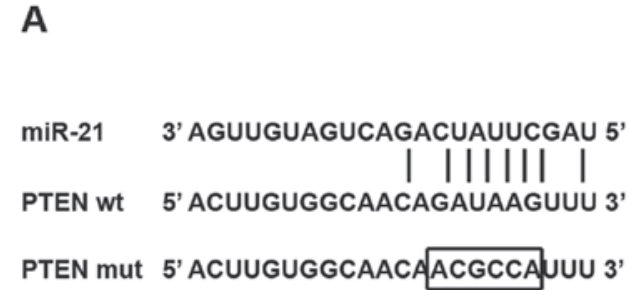

C

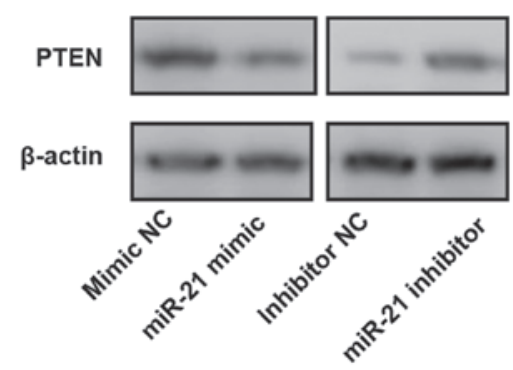

D

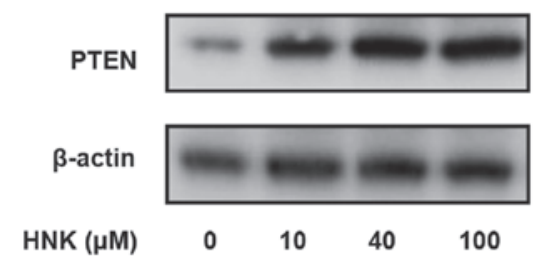

B
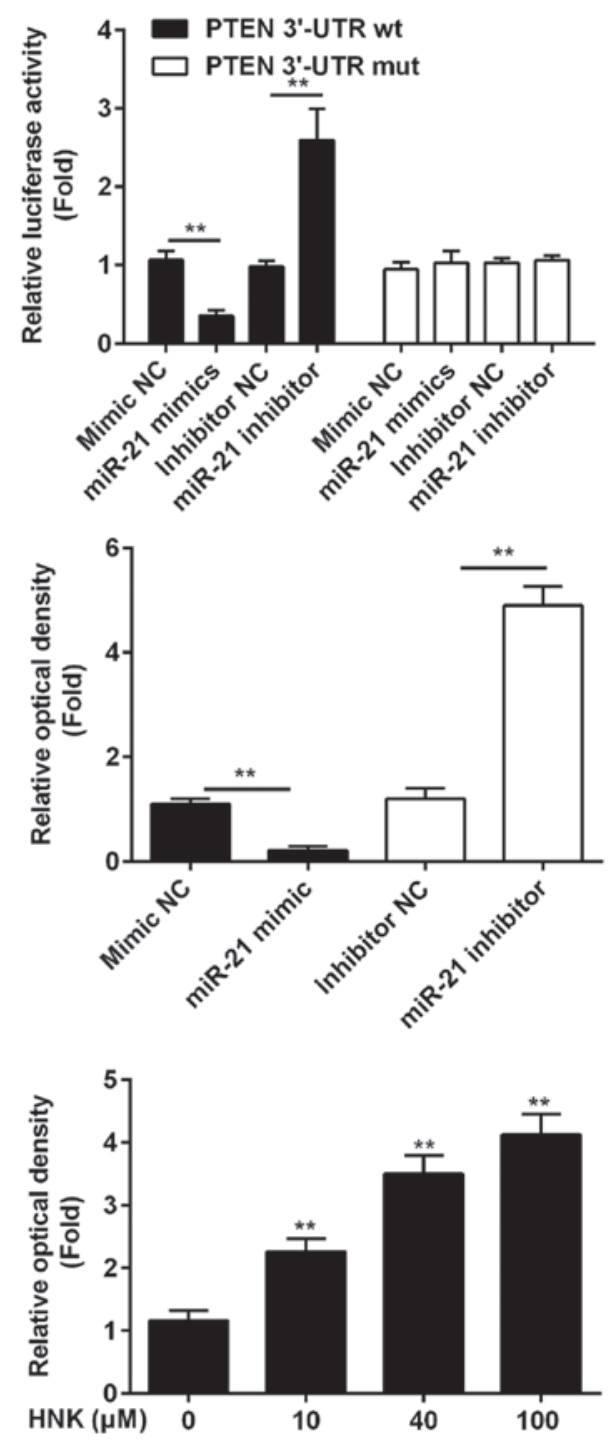

Figure 5. miR-21 suppresses PTEN expression by directly targeting its 3'-UTR. (A) PTEN 3'-UTR region containing wt or mut binding site for miR-21. (B) Relative luciferase activity of wt or mut PTEN 3'-UTR in Saos-2 cells post-transfection with miR-21 mimic/inhibitor or corresponding NC. (C) Western blot analysis was conducted to detect the protein expression levels of PTEN in Saos-2 cells post-transfection with miR-21 mimic/inhibitor or corresponding $\mathrm{NC} ; \beta$-actin was used as an internal control. (D) Saos-2 cells were treated with or without 10-100 $\mu \mathrm{M} \mathrm{HNK}$ for $24 \mathrm{~h}$, and western blot analysis was used to measure PTEN expression. Data are presented as the mean \pm standard deviation of three independent experiments. ${ }^{* *} \mathrm{P}<0.01$ vs. corresponding NC group. 3 '-UTR, 3'-untranslated region; HNK, honokiol; miR-21, microRNA-21; mut, mutant; NC, negative control; PTEN, phosphatase and tensin homolog; wt, wild-type.

signaling pathway by inhibiting miR-21 expression in human OS cells.

\section{Discussion}

HNK has been widely used to treat various diseases in traditional Chinese medicine, and it has been reported to exert anticancer functions $(9,23)$. However, it remains unclear whether HNK may be used as a stand-alone natural compound to exert strong anticancer effects against human OS. In the present study, the potential of HNK-induced apoptosis of human OS cells was examined and the underlying molecular mechanisms were investigated. The results demonstrated that HNK inhibited cell growth in a dose-dependent manner in Saos-2 and MG-63 cells. Furthermore, the results indicated that HNK-induced apoptosis was dependent upon caspase activation, and that HNK increased expression of the proapoptotic protein Bax, and reduced expression of the anti-apoptotic protein Bcl-2. Notably, the results verified that HNK may induce aberrant miRNA expression in human OS cells, and miR-21 inhibits PTEN by directly targeting its 3'-UTR. Furthermore, HNK was revealed to induce apoptosis through modulating the miR-21/PTEN/PI3K/AKT signaling pathway in human OS cells.

Natural products that are able to prevent and treat cancers are the source of numerous medically beneficial drugs, including camptothecin, curcumin, isoflavone, luteolin, matrine, HNK, phenolic leaf extract of Heimia myrtifolia (Lythraceae) and xanthoangelol $(29,30)$. A recent study demonstrated that xanthoangelol, which is isolated from Angelica keiskei roots, may inhibit tumor growth, metastasis to the lung and liver, and tumor-associated macrophage expression in tumors (30). In addition, it is well known that some natural compounds possess anticancer effects in human OS (31-33). Steinmann et al revealed that HNK exhibits prominent antimetastatic activity in OS and is able to induce rapid cell death in vitro (34). In the 
A

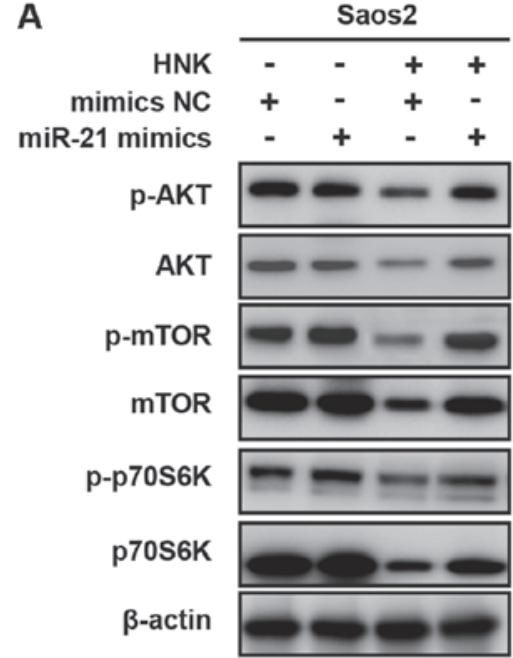

C

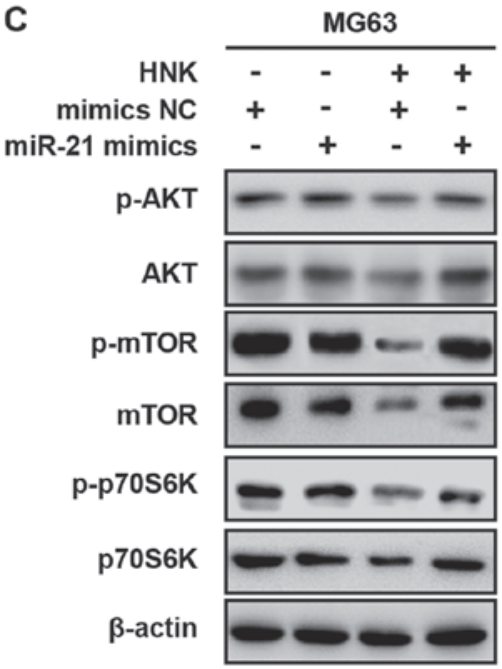

B
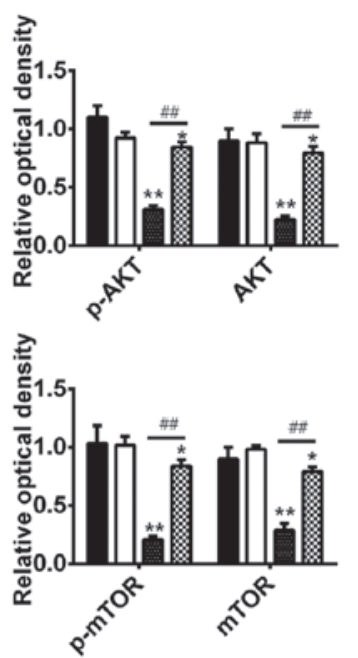

D
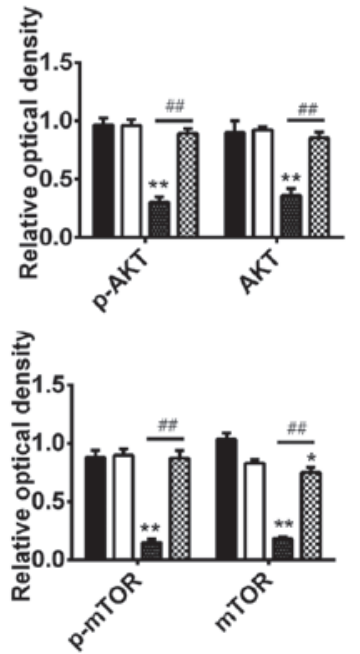

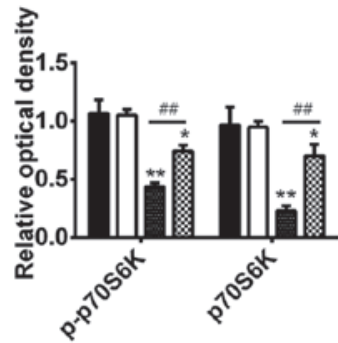

mimics NC

miR-21 mimics

HNK + mimics NC

HNK + miR-21 mimics

mimics NC

miR-21 mimics

HNK + mimics NC

界 HNK + miR-21 mimics

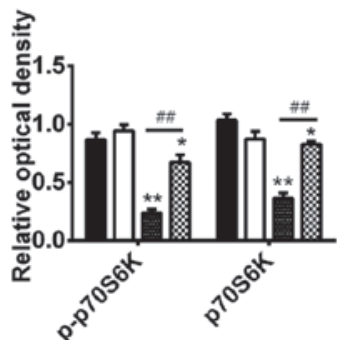

Figure 6. HNK suppresses the phosphoinositide 3-kinase/AKT signaling pathway via modulating miR-21 expression in human OS cells. Saos-2 and MG-63 cells were transfected with or without miR-21 mimics following treatment with or without HNK, and the expression levels of p-AKT, p-mTOR, p-p70S6K, AKT, mTOR and p70S6K were detected by western blot analysis in (A and B) Saos-2 and (C and D) MG-63 cells. Data are presented as the mean \pm standard deviation of three independent experiments. ${ }^{*} \mathrm{P}<0.05,{ }^{* *} \mathrm{P}<0.01$ vs. mimics $\mathrm{NC}$ group; ${ }^{\# \#} \mathrm{P}<0.01$ vs. HNK + mimics $\mathrm{NC}$ group. AKT, protein kinase $\mathrm{B}$; HNK, honokiol; miR-21, microRNA-21; mTOR, mammalian target of rapamycin; OD, optical density; OS, osteosarcoma; p70S6K, p70S6 kinase; p-, phosphorylated.

present study, the MTT assay and flow cytometry were used to determine cell viability and apoptosis in HNK-treated human OS cells; the results indicated that HNK significantly reduced cell viability and induced apoptosis of OS cells. Furthermore, it has been reported that HNK may trigger apoptosis via activation of the intrinsic or extrinsic apoptotic pathways $(9,35)$. HNK-induced apoptosis may also enhance caspase expression and PARP cleavage (23). In the present study, the results further confirmed that HNK significantly upregulated the expression levels of proapoptotic proteins (caspase-3, cleaved-PARP and Bax) and downregulated the levels of the anti-apoptotic protein Bcl-2. These data suggested that HNK may serve a crucial role in the apoptosis of OS cells.

Mounting evidence has demonstrated that miRNAs have important roles in numerous types of cancer, whereas some Chinese medicinal herbs harbor anticancer effects by targeting miRNAs. Liu et al revealed that berberine may regulate cisplatin sensitivity via mediating the miR-21/programmed cell death 4 axis in the ovarian cancer cells (18). Zeng et al demonstrated that camptothecin promotes cancer cell apoptosis via miRNA-mediated mitochondrial pathways (36). Furthermore, a recent study identified that HNK triggers the liver kinase B1-miR-34a axis and resists the oncogenic effects of leptin in breast cancer (11). Previous studies have also indicated that miR-34a modulates tumor cell growth and cell cycle progression in OS $(37,38)$. The present study revealed that HNK induced aberrant miRNA expression in a breast cancer cell line, according to microarray data obtained from the GEO database (accession no. GSE85871; http:/www.ncbi.nlm.nih. gov/geo/query/acc. cgi?acc=GSE85871). Based on these data, six miRNAs, which were most significantly upregulated or downregulated in breast cancer cells following HNK treatment, were selected and RT-qPCR was conducted to validate these results in human OS cells. The results demonstrated that miR-202 and miR-623 were significantly upregulated, whereas miR-21 was significantly downregulated in the Saos-2 human OS cell line, whereas miR-188-5p, miR-532-5p and miR-628-3p were not significantly different compared with in the vehicle group. In addition, the present study indicated that HNK treatment reduced miR-21 expression in a dose-dependent 
manner in human OS cells. Conversely, restoration of miR-21 expression abrogated the suppressive effects of HNK on OS cells. These data indicated that HNK may reduce cell viability and induce apoptosis via inhibiting miR-21 expression in human OS cells. Previous studies also reported that HNK induces apoptosis and $\mathrm{G}_{1}$ cell cycle arrest in various types of cancer cell (39-42). Furthermore, miR-21 has been identified as an important regulator of diverse cellular functions via the regulation of cancer cell growth (43-45). However, the possible molecular mechanism underlying HNK-induced apoptosis of human OS cells requires further research.

Increasing evidence has confirmed that miR-21 modulates PTEN by directly targeting its 3'-UTR in various types of cancer, including hepatocellular cancer (24) and lung cancer (46). PTEN is a tumor suppressor gene that regulates the cell cycle and apoptosis in numerous solid tumors (47). Consistent with these results, the present study confirmed that miR-21 inhibits PTEN by directly targeting its 3'-UTR in human OS cells. Datta et al revealed that the PI3K/AKT signaling pathway acts as a key oncogenic pathway to induce cell growth and survival (48), which is negatively regulated by PTEN (49). Furthermore, Bai et al reported that the PI3K/AKT signaling pathway may be modulated by miR-21 in hepatocytes (50). The present study revealed that HNK suppresses the PI3K/AKT signaling pathway; however, it could be reactivated by miR-21 overexpression. Taken together, these data indicated that HNK induced apoptosis of human OS cells via modulating the miR-21/PTEN/PI3K/AKT signaling pathway.

In conclusion, the present study provided a novel insight into the molecular mechanism underlying HNK-induced apoptosis of human OS cells. Notably, the present results confirmed that HNK induces aberrant miRNA expression and HNK-induced apoptosis was modulated by the miR-21/PTEN/PI3K/AKT signaling pathway in human OS cells. These results suggested that HNK may exert anticancer effects to prevent OS progression.

\section{Competing interests}

The authors declare there is no competing interest.

\section{References}

1. Hung GY, Horng JL, Yen HJ, Yen CC, Chen WM, Chen PC, Wu HT and Chiou HJ: Incidence patterns of primary bone cancer in taiwan (2003-2010): A population-based study. Ann Surg Oncol 21: 2490-2498, 2014.

2. He H, Ni J and Huang J: Molecular mechanisms of chemoresistance in osteosarcoma (Review). Oncol Lett 7: 1352-1362, 2014.

3. Longhi A, Errani C, De Paolis M, Mercuri M and Bacci G: Primary bone osteosarcoma in the pediatric age: State of the art. Cancer Treat Rev 32: 423-436, 2006.

4. Marina N, Gebhardt M, Teot L and Gorlick R: Biology and therapeutic advances for pediatric osteosarcoma. Oncologist 9: 422-441, 2004

5. Steinmann P, Walters DK, Arlt MJ, Banke IJ,Ziegler U,Langsam B, Arbiser J, Muff R, Born W and Fuchs B: Antimetastatic activity of honokiol in osteosarcoma. Cancer 118: 2117-2127, 2012.

6. Ho KY, Tsai CC, Chen CP, Huang JS and Lin CC: Antimicrobial activity of honokiol and magnolol isolated from Magnolia officinalis. Phytother Res 15: 139-141, 2001.

7. Ou HC, Chou FP, Lin TM, Yang CH and Sheu WH: Protective effects of honokiol against oxidized LDL-induced cytotoxicity and adhesion molecule expression in endothelial cells. Chem Biol Interact 161: 1-13, 2006.
8. Bai X, Cerimele F, Ushio-Fukai M, Waqas M, Campbell PM, Govindarajan B, Der CJ, Battle T, Frank DA, Ye K, et al: Honokiol, a small molecular weight natural product, inhibits angiogenesis in vitro and tumor growth in vivo. J Biol Chem 278: 35501-35507, 2003.

9. Ishitsuka K, Hideshima T, Hamasaki M, Raje N, Kumar S, Hideshima H, Shiraishi N, Yasui H, Roccaro AM, Richardson P, et al: Honokiol overcomes conventional drug resistance in human multiple myeloma by induction of caspase-dependent and -independent apoptosis. Blood 106: 1794-1800, 2005.

10. Chen F, Wang T, Wu Y-F, Gu Y, Xu X-L, Zheng S and Hu X: Honokiol: A potent chemotherapy candidate for human colorectal carcinoma. World J Gastroenterol 10: 3459-3463, 2004.

11. Avtanski DB, Nagalingam A, Kuppusamy P, Bonner MY, Arbiser JL, Saxena NK and Sharma D: Honokiol abrogates leptin-induced tumor progression by inhibiting Wnt1-MTA1- $\beta$-catenin signaling axis in a microRNA-34a dependent manner. Oncotarget 6: 16396-16410, 2015.

12. Sheu ML,Liu SH and Lan KH:Honokiol induces calpain-mediated glucose-regulated protein-94 cleavage and apoptosis in human gastric cancer cells and reduces tumor growth. PLoS One 2: e1096, 2007.

13. Chen YJ, Wu CL, Liu JF, Fong YC, Hsu SF, Li TM, Su YC, Liu SH and Tang CH: Honokiol induces cell apoptosis in human chondrosarcoma cells through mitochondrial dysfunction and endoplasmic reticulum stress. Cancer Lett 291: 20-30, 2010.

14. Hahm ER and Singh SV: Honokiol causes G0-G1 phase cell cycle arrest in human prostate cancer cells in association with suppression of retinoblastoma protein level/phosphorylation and inhibition of E2F1 transcriptional activity. Mol Cancer Ther 6: 2686-2695, 2007.

15. Crane C, Panner A, Pieper RO, Arbiser J and Parsa AT: Honokiol mediated inhibition of PI3K/mTOR pathway: A potential strategy to overcome immunoresistance in glioma, breast and prostate carcinoma without impacting $\mathrm{T}$ cell function. J Immunother 32 : 585-592, 2009.

16. Croce CM: Causes and consequences of microRNA dysregulation in cancer. Nat Rev Genet 10: 704-714, 2009.

17. Zamani $M$, Sadeghizadeh $M$, Behmanesh $M$ and Najafi F: Dendrosomal curcumin increases expression of the long non-coding RNA gene MEG3 via up-regulation of epi-miRs in hepatocellular cancer. Phytomedicine 22: 961-967, 2015.

18. Liu S, Fang Y, Shen H, Xu W and Li H: Berberine sensitizes ovarian cancer cells to cisplatin through miR-21/PDCD4 axis. Acta Biochim Biophys Sin (Shanghai) 45: 756-762, 2013.

19. Hong M, Wang N, Tan HY, Tsao SW and Feng Y: MicroRNAs and Chinese Medicinal Herbs: New possibilities in cancer therapy. Cancers (Basel) 7: 1643-1657,2015.

20. Zhang Q, Zhao W, Ye C, Zhuang J, Chang C, Li Y, Huang X, Shen L, Li Y, Cui Y, et al: Honokiol inhibits bladder tumor growth by suppressing EZH2/miR-143 axis. Oncotarget 6: 37335-37348, 2015.

21. Lai YJ, Lin CI, Wang CL and Chao JI: Expression of survivin and p53 modulates honokiol-induced apoptosis in colorectal cancer cells. J Cell Biochem 115: 1888-1899, 2014.

22. Livak KJ and Schmittgen TD: Analysis of relative gene expression data using real-time quantitative PCR and the 2(-Delta Delta C(T)) Method. Methods 25: 402-408, 2001.

23. Battle TE, Arbiser J and Frank DA: The natural product honokiol induces caspase-dependent apoptosis in B-cell chronic lymphocytic leukemia (B-CLL) cells. Blood 106: 690-697, 2005.

24. Meng F, Henson R, Wehbe-Janek H, Ghoshal K, Jacob ST and Patel T: MicroRNA-21 regulates expression of the PTEN tumor suppressor gene in human hepatocellular cancer. Gastroenterology 133: 647-658, 2007.

25. Wu YR, Qi HJ, Deng DF, Luo YY and Yang SL: MicroRNA-21 promotes cell proliferation, migration, and resistance to apoptosis through PTEN/PI3K/AKT signaling pathway in esophageal cancer. Tumour Biol 37: 12061-12070, 2016.

26. Liu ZL, Wang H, Liu J and Wang ZX: MicroRNA-21 (miR-21) expression promotes growth, metastasis, and chemo- or radioresistance in non-small cell lung cancer cells by targeting PTEN. Mol Cell Biochem 372: 35-45, 2013.

27. Guo H, German P, Bai S, Barnes S, Guo W, Qi X, Lou H, Liang J, Jonasch E, Mills GB, et al: The PI3K/AKT pathway and renal cell carcinoma. J Genet Genomics 42: 343-353, 2015.

28. Fang Y, Xue JL, Shen Q, Chen J and Tian L: MicroRNA-7 inhibits tumor growth and metastasis by targeting the phosphoinositide 3-kinase/Akt pathway in hepatocellular carcinoma. Hepatology 55: 1852-1862, 2012. 
29. Ayoub N, Singab AN, El-Naggar M and Lindequist U: Investigation of phenolic leaf extract of Heimia myrtifolia (Lythraceae): Pharmacological properties (stimulation of mineralization of SaOS-2 osteosarcoma cells) and identification of polyphenols. Drug Discov Ther 4: 341-348, 2010.

30. Sumiyoshi M, Taniguchi M, Baba K and Kimura Y: Antitumor and antimetastatic actions of xanthoangelol and 4-hydroxyderricin isolated from Angelica keiskei roots through the inhibited activation and differentiation of M2 macrophages. Phytomedicine 22: 759-767, 2015.

31. Xia YZ, Ni K, Guo C, Zhang C, Geng YD, Wang ZD, Yang L and Kong LY: Alopecurone B reverses doxorubicin-resistant human osteosarcoma cell line by inhibiting P-glycoprotein and NF-kappa B signaling. Phytomedicine 22: 344-351, 2015.

32. Wang T, Gong X, Jiang R, Li H, Du W and Kuang G: Ferulic acid inhibits proliferation and promotes apoptosis via blockage of PI3K/Akt pathway in osteosarcoma cell. Am J Transl Res 8 968-980, 2016.

33. Er S and Dikmen M: Camellia sinensis increased apoptosis on $\mathrm{U}_{2} \mathrm{OS}$ osteosarcoma cells and wound healing potential on $\mathrm{NIH}_{3} \mathrm{~T}_{3}$ fibroblast cells. Cytotechnology: May 16, 2017 (Epub ahead of print). doi: 10.1007/s10616-017-0105-4.

34. Steinmann P, Walters DK, Arlt MJ, Banke IJ, Ziegler U, Langsam B, Arbiser J, Muff R, Born W and Fuchs B: Antimetastatic activity of honokiol in osteosarcoma. Cancer 118: 2117-2127.

35. Raja SM, Chen S, Yue P, Acker TM, Lefkove B, Arbiser JL, Khuri FR and Sun SY: The natural product honokiol preferentially inhibits cellular FLICE-inhibitory protein and augments death receptor-induced apoptosis. Mol Cancer Ther 7: 2212-2223, 2008.

36. Zeng CW, Zhang XJ, Lin KY, Ye H, Feng SY, Zhang H and Chen YQ: Camptothecin induces apoptosis in cancer cells via microRNA-125b-mediated mitochondrial pathways. Mol Pharmacol 81: 578-586, 2012.

37. Novello C, Pazzaglia L, Conti A, Quattrini I, Pollino S, Perego P, Picci P and Benassi MS: p53-dependent activation of microRNA-34a in response to etoposide-induced DNA damage in osteosarcoma cell lines not impaired by dominant negative p53 expression. PLoS One 9: e114757, 2014.

38. Zhao Y, Tu MJ, Yu YF, Wang WP, Chen QX, Qiu JX, Yu AX and Yu AM: Combination therapy with bioengineered miR-34a prodrug and doxorubicin synergistically suppresses osteosarcoma growth. Biochem Pharmacol 98: 602-613, 2015.

39. Lin CJ, Chang YA, Lin YL, Liu SH, Chang CK and Chen RM: Preclinical effects of honokiol on treating glioblastoma multiforme via G1 phase arrest and cell apoptosis. Phytomedicine 23: 517-527, 2016.
40. Luo LX, Li Y,Liu ZQ, Fan XX, Duan FG,Li RZ, Yao XJ,Leung EL and Liu L: Honokiol induces apoptosis, G1 arrest, and autophagy in KRAS mutant lung cancer cells. Front Pharmacol 8: 199, 2017.

41. Chen HC, Hsu HT, Weng JW, Chang YF, Hsia CY, Lee HC and Chi CW: Combined effect of honokiol and rosiglitazone on cell growth inhibition through enhanced G0/G1 phase arrest in hepatoma cells. J Chin Med Assoc 79: 415-421, 2016.

42. Benhamouche-Trouillet S and Postic C: Emerging role of miR-2 in non-alcoholic fatty liver disease. Gut 65: 1781-1783, 2016.

43. Xu B, Xia H, Cao J, Wang Z, Yang Y and Lin Y: MicroRNA-21 inhibits the apoptosis of osteosarcoma cell line SAOS-2 via targeting caspase-8. Oncol Res: Jan 20, 2017 (Epub ahead of print). doi: 10.3727/096504017X14841698396829.

44. Wu YR, Qi HJ, Deng DF, Luo YY and Yang SL: MicroRNA-21 promotes cell proliferation, migration, and resistance to apoptosis through PTEN/PI3K/AKT signaling pathway in esophageal cancer. Tumour Biol 37: 12061-12070, 2016.

45. Lv C, Hao Y and Tu G: MicroRNA-21 promotes proliferation, invasion and suppresses apoptosis in human osteosarcoma line MG63 through PTEN/Akt pathway. Tumour Biol 37: 9333-9342, 2016.

46. Yang Z, Fang S, Di Y, Ying W, Tan Y and Gu W: Modulation of NF- $\mathrm{BB} / \mathrm{miR}-21 / \mathrm{PTEN}$ pathway sensitizes non-small cell lung cancer to cisplatin. PLoS One 10: e0121547, 2015.

47. Xu LF, Wu ZP, Chen Y, Zhu QS, Hamidi S and Navab R: MicroRNA-21 (miR-21) regulates cellular proliferation, invasion, migration, and apoptosis by targeting PTEN, RECK and Bcl-2 in lung squamous carcinoma, Gejiu City, China. PLoS One 9: e103698, 2014

48. Datta SR, Brunet A and Greenberg ME: Cellular survival: A play in three Akts. Genes Dev 13: 2905-2927, 1999.

49. Di Cristofano A and Pandolfi PP: The multiple roles of PTEN in tumor suppression. Cell 100: 387-390, 2000.

50. Bai YN, Yu ZY, Luo LX, Yi J, Xia QJ and Zeng Y: MicroRNA-21 accelerates hepatocyte proliferation in vitro via PI3K/Akt signaling by targeting PTEN. Biochem Biophys Res Commun 443: 802-807, 2014.

This work is licensed under a Creative Commons Attribution-NonCommercial-NoDerivatives 4.0 International (CC BY-NC-ND 4.0) License. 